\title{
Scheme variations of the QCD coupling
}

\author{
Diogo Boito ${ }^{1}$, Matthias Jamin ${ }^{2,3, a}$, and Ramon Miravitllas ${ }^{2}$ \\ ${ }^{1}$ Instituto de Física de São Carlos, Universidade de São Paulo, CP 369, 13560-970, São Carlos, SP, Brazil \\ ${ }^{2}$ IFAE, BIST, Campus UAB, 08193 Bellaterra (Barcelona) Spain \\ ${ }^{3}$ ICREA, Pg. Lluís Companys 23, 08010 Barcelona, Spain
}

\begin{abstract}
The Quantum Chromodynamics (QCD) coupling $\alpha_{s}$ is a central parameter in the Standard Model of particle physics. However, it depends on theoretical conventions related to renormalisation and hence is not an observable quantity. In order to capture this dependence in a transparent way, a novel definition of the QCD coupling, denoted by $\hat{a}$, is introduced, whose running is explicitly renormalisation scheme invariant. The remaining renormalisation scheme dependence is related to transformations of the QCD scale $\Lambda$, and can be parametrised by a single parameter $C$. Hence, we call $\hat{a}$ the $C$-scheme coupling. The dependence on $C$ can be exploited to study and improve perturbative predictions of physical observables. This is demonstrated for the QCD Adler function and hadronic decays of the $\tau$ lepton.
\end{abstract}

\section{Introduction}

A central approach to predictions in Quantum Chromodynamics (QCD) relies on perturbation theory in the strong coupling $\alpha_{s}$. However, quarks do not appear as free particles in nature, they are confined into hadrons, and the definition of $\alpha_{s}$ depends on theoretical conventions like the renormalisation scale or renormalisation scheme. Evidently, physical observables should not depend on such choices, which is reflected in so-called renormalisation group equations (RGE's) for scale variations, which measurable quantities have to satisfy. The situation regarding the scheme dependence is more involved because the strong coupling can be redefined order by order in perturbation theory. For this reason, perturbative computations are performed mainly in convenient schemes such as minimal subtraction (MS) [1] or modified minimal subtraction ( $\overline{\mathrm{MS}})$ [2].

This talk reports on a recent work [3], in which a new definition of the strong coupling, $\hat{a}$, was introduced. The coupling $\hat{a}$ satisfies two nice properties: first, its scale evolution, described by the $\beta$-function, is explicitly scheme invariant. Second, the remaining scheme dependence of $\hat{a}$ can be parametrised by a single parameter $C$. For this reason, henceforth, we shall refer to $\hat{a}$ as the $C$-scheme coupling, even though $C$ parametrises a whole class of schemes. Variations of $C$ directly correspond to transformations of the QCD scale parameter $\Lambda$. Furthermore, it can be demonstrated that the $C$ dependence of $\hat{a}$ is also governed by the corresponding $\beta$-function.

After an introduction of the coupling $\hat{a}$ and the discussion of its properties, we proceed to apply it to phenomenologically relevant cases. One of the best studied QCD quantities is the two-point vector

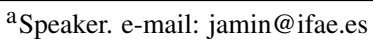


correlation function and its derivative, the Adler function [4], which is related to a spectral integral over the total cross section for $e^{+} e^{-}$scattering into hadrons. It also governs theoretical predictions of the inclusive decay rate of $\tau$ leptons into hadronic final states [5]. Presently, the perturbative series for the Adler function is known up to the fourth order in $\alpha_{s}$ [6]. Exploiting the scheme dependence of the coupling $\hat{a}$ through variations of the parameter $C$, it can be shown how to improve theoretical predictions for the phenomenological quantities. The use of $\hat{a}$ for the scalar correlator, which is relevant for the prediction of Higgs boson decay into quarks and for light quark-mass determinations from QCD sum rules, has been investigated in a related article [7].

In the past, several other methods have been suggested to optimise perturbative predictions. In such approaches like BLM [8], or the Principle of Maximum Conformality (PMC) [9, 10], either a scale-setting prescription is provided to obtain a scheme-independent result, regardless of the intermediate scheme used for the perturbative calculation (which most often is $\overline{\mathrm{MS}}$ ). On the other hand, some of these approaches, such as for example the "effective charge" [11], involve a process dependent definition of the QCD coupling. On the contrary, in the procedure discussed here, one defines a process-independent class of schemes, parameterised by a single continuous parameter $C$. Variations of this parameter are then explored in order to optimise the perturbative series having in mind that we are dealing with asymptotic expansions. Preferred values of the parameter $C$, however, may then depend on the quantity under consideration.

\section{The QCD coupling $\hat{\alpha}_{s}$}

To begin, we define the QCD $\beta$-function, which describes the scale evolution of the coupling $\alpha_{s}$, as

$$
-Q \frac{\mathrm{d} a_{Q}}{\mathrm{~d} Q} \equiv \beta\left(a_{Q}\right)=\beta_{1} a_{Q}^{2}+\beta_{2} a_{Q}^{3}+\beta_{3} a_{Q}^{4}+\ldots
$$

Here and in the following, we use the abbreviation $a_{Q} \equiv \alpha_{s}(Q) / \pi$, and $Q$ denotes a physically relevant energy scale. The first five $\beta$-coefficients $\beta_{1}$ to $\beta_{5}$ are known analytically $[12,13]$. (In the conventions employed in this work, they have been collected in Appendix A of ref. [7].) Making use of the RGE (1) for $a_{Q}$, the scale-invariant QCD parameter $\Lambda$ can be defined by

$$
\Lambda \equiv Q \mathrm{e}^{-\frac{1}{\beta_{1} a} Q}\left[a_{Q}\right]^{-\frac{\beta_{2}}{\beta_{1}^{2}}} \exp \left\{\int_{0}^{a_{Q}} \frac{\mathrm{d} a}{\tilde{\beta}(a)}\right\},
$$

with the combination

$$
\frac{1}{\tilde{\beta}(a)} \equiv \frac{1}{\beta(a)}-\frac{1}{\beta_{1} a^{2}}+\frac{\beta_{2}}{\beta_{1}^{2} a},
$$

which remains free of singularities in the limit $a \rightarrow 0$. Let us consider a scheme transformation to another coupling $a^{\prime}$, which assumes the general form

$$
a^{\prime} \equiv a+c_{1} a^{2}+c_{2} a^{3}+c_{3} a^{4}+\ldots
$$

The $\Lambda$-parameter in the transformed scheme, $\Lambda^{\prime}$, depends only on $c_{1}$ and not on the remaining higherorder coefficients. The actual relation reads [14]

$$
\Lambda^{\prime}=\Lambda \mathrm{e}^{c_{1} / \beta_{1}} .
$$


The observation that redefinitions of the $\Lambda$-parameter only involve a single constant motivates an implicit definition of a new coupling $\hat{a}_{Q}$, which is scheme invariant except for shifts in $\Lambda$, represented by a parameter $C[3]$ :

$$
\frac{1}{\hat{a}_{Q}}+\frac{\beta_{2}}{\beta_{1}} \ln \hat{a}_{Q}-\frac{\beta_{1}}{2} C \equiv \beta_{1} \ln \frac{Q}{\Lambda}=\frac{1}{a_{Q}}+\frac{\beta_{2}}{\beta_{1}} \ln a_{Q}-\beta_{1} \int_{0}^{a_{Q}} \frac{\mathrm{d} a}{\tilde{\beta}(a)} .
$$

In perturbation theory, eq. (6) should be interpreted in an iterative sense. Obviously, $\hat{a}_{Q}$ is a function of $C$ but, for notational simplicity, this dependence will not be made explicit. It should be remarked that a combination similar to (6), but without the logarithmic term on the left-hand side, has already been discussed in refs. $[15,16]$. Without this term, however, an unwelcome logarithm of $a_{Q}$ remains in the perturbative relation between the couplings $\hat{a}_{Q}$ and $a_{Q}$. This non-analytic contribution is avoided by the construction of eq. (6). In fig. 1, the coupling $\hat{a}$ according to eq. (6) is displayed as a function of $C$. Since in this work we focus on hadronic $\tau$ decays, our initial $\overline{\mathrm{MS}}$ input is employed as $\alpha_{s}\left(M_{\tau}\right)=$ $0.316(10)$, which results from the current PDG average $\alpha_{s}\left(M_{Z}\right)=0.1181(13)$ [17]. The yellow band corresponds to the variation within present $\alpha_{s}$ uncertainties. Below approximately $C=-2$, the relation between $\hat{a}$ and the $\overline{\mathrm{MS}}$ coupling ceases to be perturbative and breaks down.

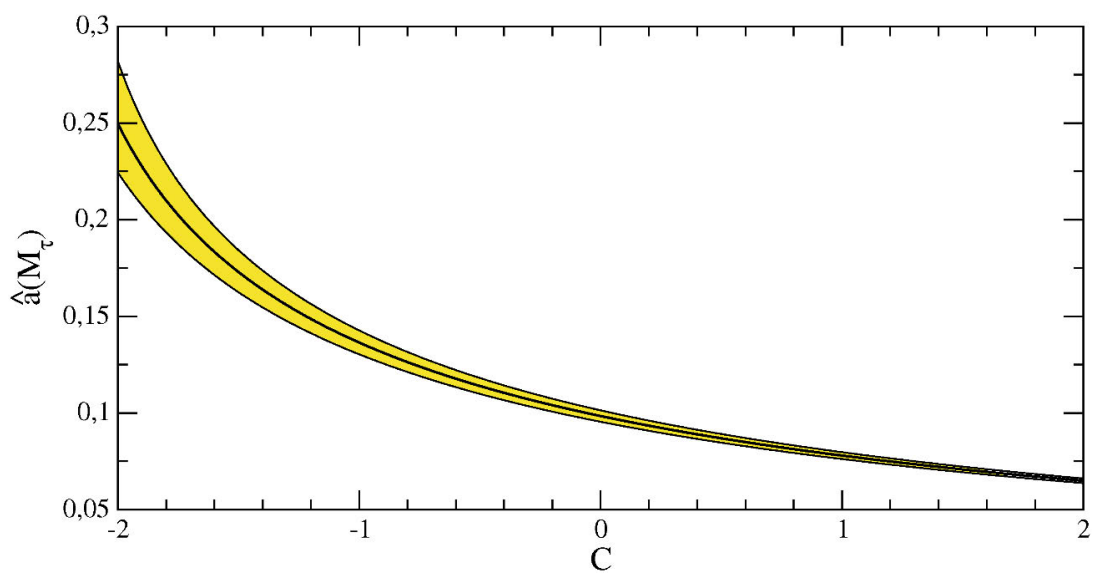

Figure 1. The coupling $\hat{a}\left(M_{\tau}\right)$ according to eq. (6) as a function of $C$, and for the input value $\alpha_{s}\left(M_{\tau}\right)=0.316(10)$ in the $\overline{\mathrm{MS}}$ scheme. The yellow band corresponds to the $\alpha_{s}$ uncertainty.

As a next step, the $\beta$-function and the corresponding running of the coupling $\hat{a}$ can be deduced from eq. (6). The $\beta$-function is found to have the rather simple form

$$
-Q \frac{\mathrm{d} \hat{a}_{Q}}{\mathrm{~d} Q} \equiv \hat{\beta}\left(\hat{a}_{Q}\right)=\frac{\beta_{1} \hat{a}_{Q}^{2}}{\left(1-\frac{\beta_{2}}{\beta_{1}} \hat{a}_{Q}\right)} .
$$

As is seen explicitly, it only depends on the scheme-invariant $\beta$-function coefficients $\beta_{1}$ and $\beta_{2}$. We also remark that the only non-trivial zero of $\hat{\beta}(\hat{a})$ arises in the case of $\beta_{1}=0$. Straightforward integration of the RGE (7) yields

$$
\frac{1}{\hat{a}_{Q}}=\frac{1}{\hat{a}_{\mu}}+\frac{\beta_{1}}{2} \ln \frac{Q^{2}}{\mu^{2}}-\frac{\beta_{2}}{\beta_{1}} \ln \frac{\hat{a}_{Q}}{\hat{a}_{\mu}} .
$$


This implicit equation for $\hat{a}_{Q}$ can either be solved numerically, or iteratively, to provide a perturbative expansion. The evolution in $C$ can also be expressed in terms of an RGE. Simply taking the derivative of eq. (6) with respect to $C$, one derives the relation

$$
\frac{\mathrm{d} \hat{a}_{Q}}{\mathrm{~d} C}=-\frac{1}{2} \hat{\beta}\left(\hat{a}_{Q}\right)
$$

demonstrating that the $C$-"running" is also governed by the $\beta$-function $\hat{\beta}\left(\hat{a}_{Q}\right)$. This explains why the $C$ dependence of $\hat{a}\left(M_{\tau}\right)$ displayed in fig. 1 appears similar to the scale running. They are in fact equivalent.

The latter observation opens the possibility to arrive at the perturbative expansion in the $C$-scheme at arbitrary $C$ by first computing the expansion in $\hat{a}_{Q}$ at $C=0$, and then employing the evolution equation (9) to arrive at an arbitrary $C$. This is completely analogous to the possibility of reconstructing the scale logarithms from the RGE in the renormalisation scale. Let us define the abbreviation $\bar{a}_{Q} \equiv \hat{a}_{Q}^{C=0}$. Then the relation between the coupling $a_{Q}$ and its corresponding $\beta$-function coefficients in an arbitrary scheme, for example the $\overline{\mathrm{MS}}$ scheme, and $\bar{a}_{Q}$ is found to be

$$
\begin{aligned}
a_{Q}=\bar{a}_{Q} & +\left(\frac{\beta_{3}}{\beta_{1}}-\frac{\beta_{2}^{2}}{\beta_{1}^{2}}\right) \bar{a}_{Q}^{3}+\left(\frac{\beta_{4}}{2 \beta_{1}}-\frac{\beta_{2}^{3}}{2 \beta_{1}^{3}}\right) \bar{a}_{Q}^{4} \\
& +\left(\frac{\beta_{5}}{3 \beta_{1}}-\frac{\beta_{2} \beta_{4}}{6 \beta_{1}^{2}}+\frac{5 \beta_{3}^{2}}{3 \beta_{1}^{2}}-\frac{3 \beta_{2}^{2} \beta_{3}}{\beta_{1}^{3}}+\frac{7 \beta_{2}^{4}}{6 \beta_{1}^{4}}\right) \bar{a}_{Q}^{5}+O\left(\bar{a}_{Q}^{6}\right) .
\end{aligned}
$$

The successive relation between the coupling $\hat{a}_{Q}$ at arbitrary $C$ and $\bar{a}_{Q}$ which only contains the scheme-invariant coefficients $\beta_{1}$ and $\beta_{2}$, can then be derived from the RGE (9). It is found to take the form

$$
\begin{aligned}
\bar{a}_{Q}=\hat{a}_{Q} & +\frac{\beta_{1}}{2} C \hat{a}_{Q}^{2}+\left(\frac{\beta_{2}}{2} C+\frac{\beta_{1}^{2}}{4} C^{2}\right) \hat{a}_{Q}^{3}+\left(\frac{\beta_{2}^{2}}{2 \beta_{1}} C+\frac{5 \beta_{1} \beta_{2}}{8} C^{2}+\frac{\beta_{1}^{3}}{8} C^{3}\right) \hat{a}_{Q}^{4} \\
& +\left(\frac{\beta_{2}^{3}}{2 \beta_{1}^{2}} C+\frac{9 \beta_{2}^{2}}{8} C^{2}+\frac{13 \beta_{1}^{2} \beta_{2}}{24} C^{3}+\frac{\beta_{1}^{4}}{16} C^{4}\right) \hat{a}_{Q}^{5}+O\left(\hat{a}_{Q}^{6}\right)
\end{aligned}
$$

Inserting now eq. (11) into (10), the perturbative relations between the coupling $\hat{a}$ and $a$ in a particular scheme can straightforwardly be deduced. Taking $a$ as well as the respective $\beta$-function coefficients in the $\overline{\mathrm{MS}}$ scheme, and for three quark flavors, $N_{f}=3$, the expansions read,

$$
\begin{aligned}
a(\hat{a})=\hat{a} & +\frac{9}{4} C \hat{a}^{2}+\left(\frac{3397}{2592}+4 C+\frac{81}{16} C^{2}\right) \hat{a}^{3}+\left(\frac{741103}{186624}+\frac{18383}{1152} C+\frac{45}{2} C^{2}\right. \\
& \left.+\frac{729}{64} C^{3}+\frac{445}{144} \zeta_{3}\right) \hat{a}^{4}+\left(\frac{1142666849}{80621568}+\frac{1329359}{20736} C+\frac{28623}{256} C^{2}+\frac{351}{4} C^{3}\right. \\
& \left.+\frac{6561}{256} C^{4}+\frac{445}{16} \zeta_{3} C+\frac{10375693}{373248} \zeta_{3}-\frac{1335}{256} \zeta_{4}-\frac{534385}{20736} \zeta_{5}\right) \hat{a}^{5}+O\left(\hat{a}^{6}\right),
\end{aligned}
$$

and

$$
\begin{aligned}
\hat{a}(a)=a & -\frac{9}{4} C a^{2}-\left(\frac{3397}{2592}+4 C-\frac{81}{16} C^{2}\right) a^{3}-\left(\frac{741103}{186624}+\frac{233}{192} C-\frac{45}{2} C^{2}\right. \\
& \left.+\frac{729}{64} C^{3}+\frac{445}{144} \zeta_{3}\right) a^{4}-\left(\frac{727240925}{80621568}-\frac{869039}{41472} C-\frac{26673}{512} C^{2}+\frac{351}{4} C^{3}\right. \\
& \left.-\frac{6561}{256} C^{4}-\frac{445}{32} \zeta_{3} C+\frac{10375693}{373248} \zeta_{3}-\frac{1335}{256} \zeta_{4}-\frac{534385}{20736} \zeta_{5}\right) a^{5}+O\left(a^{6}\right),
\end{aligned}
$$


where $\zeta_{i} \equiv \zeta(i)$ stands for the Riemann $\zeta$-function. Eqs. (12) and (13) have originally been presented in ref. [3].

\section{The Adler function}

As our first application of the coupling $\hat{\alpha}_{s}$, the perturbative series of the Adler function $D\left(a_{Q}\right)[4,6]$ shall be investigated. To this end, it is convenient to define the reduced Adler function $\hat{D}\left(a_{Q}\right)$ as

$$
4 \pi^{2} D\left(a_{Q}\right)-1 \equiv \hat{D}\left(a_{Q}\right)=\sum_{n=1}^{\infty} c_{n, 1} a_{Q}^{n}=a_{Q}+1.640 a_{Q}^{2}+6.371 a_{Q}^{3}+49.08 a_{Q}^{4}+\ldots
$$

We adopt the notation of ref. [18], with numerical coefficients at $N_{f}=3$ and in the $\overline{\mathrm{MS}}$ scheme. The renormalisation scale logarithms $\ln (Q / \mu)$ appearing in the Adler function have been resummed with the scale choice $\mu=Q$.

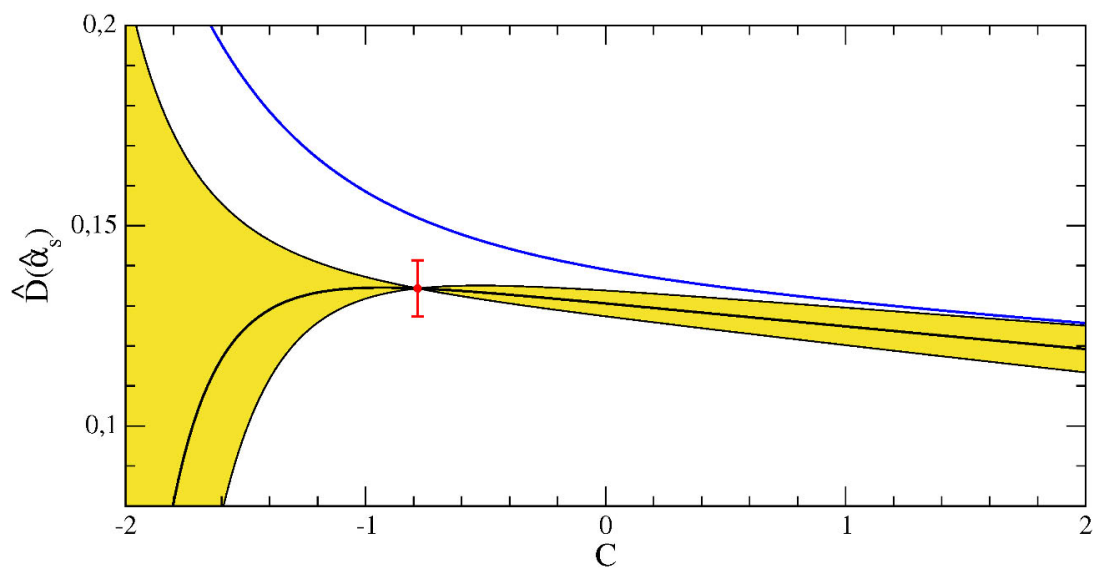

Figure 2. $\hat{D}\left(\hat{a}_{M_{\tau}}\right)$ of eq. (15) as a function of $C$. The yellow band arises from either removing or doubling the fifth-order term. In the red dot, the $O\left(\hat{a}^{5}\right)$ vanishes, and $O\left(\hat{a}^{4}\right)$ is taken as the uncertainty. For further explanation, see the text.

Employing the relation (12), the expansion (14) for $\hat{D}$ can be rewritten in terms of the $C$-scheme coupling $\hat{a}_{Q}$, resulting in

$$
\begin{aligned}
\hat{D}\left(\hat{a}_{Q}\right)= & \hat{a}_{Q}+(1.640+2.25 C) \hat{a}_{Q}^{2}+\left(7.682+11.38 C+5.063 C^{2}\right) \hat{a}_{Q}^{3} \\
& +\left(61.06+72.08 C+47.40 C^{2}+11.39 C^{3}\right) \hat{a}_{Q}^{4}+\ldots
\end{aligned}
$$

A graphical representation of eq. (15) is provided in Fig. 2, where $\hat{D}\left(\hat{a}_{M_{\tau}}\right)$ is displayed as a function of the scheme parameter $C$. Here, the yellow band represents an error estimate from the fifth-order contribution. The required coefficient was taken to be $c_{5,1}=283$, according to an estimate deduced in ref. [18]. The yellow band then corresponds to either removing or doubling the $O\left(\hat{a}^{5}\right)$ term. Generally, it is observed that around $C \approx-1$, a region of stability with respect to $C$-variations emerges. For comparison, the blue line corresponds to employing $c_{5,1}=566$ and in addition doubling the $O\left(\hat{a}^{5}\right)$ correction. Then, no stability is found which seems to indicate that such large values of $c_{5,1}$ are disfavoured. In the red dot, where $C=-0.783$, the $O\left(\hat{a}^{5}\right)$ vanishes, and the $O\left(\hat{a}^{4}\right)$ correction has been 
employed as a conservative uncertainty, which is the last included non-vanishing term, in view of the asymptotic nature of the series. Numerically, it reads

$$
\hat{D}\left(\hat{a}_{M_{\tau}}, C=-0.783\right)=0.1343 \pm 0.0070 \pm 0.0067,
$$

where the second error originates from the uncertainty in $\alpha_{s}\left(M_{\tau}\right)$. The result (16) can be compared to the direct $\overline{\mathrm{MS}}$ prediction (14), which corresponds to

$$
\hat{D}\left(a_{M_{\tau}}\right)=0.1316 \pm 0.0029 \pm 0.0060 .
$$

Here, the first error is obtained by removing or doubling $c_{5,1}$, and the second error again reflects the parametric $\alpha_{s}$ uncertainty.

A final comparison of (16) and (17) may be performed with the Adler function model that was put forward in ref. [18], and which is based on general knowledge of the renormalon structure for the Borel transform of $\hat{D}\left(a_{Q}\right)$. Within this model, one obtains

$$
\hat{D}\left(a_{M_{\tau}}\right)=0.1354 \pm 0.0127 \pm 0.0058 .
$$

In this case, the first uncertainty results from estimates of the perturbative ambiguity that arises from the renormalon singularities. It is observed that this uncertainty is substantially bigger than the one of (17) and still larger than the one of (16). Therefore, we conclude that the higher-order uncertainty of (17) appears to be underestimated, while eq. (16) seems to provide a more realistic account of the resummed series. Interestingly enough, also its central value is closer to the Borel model result.

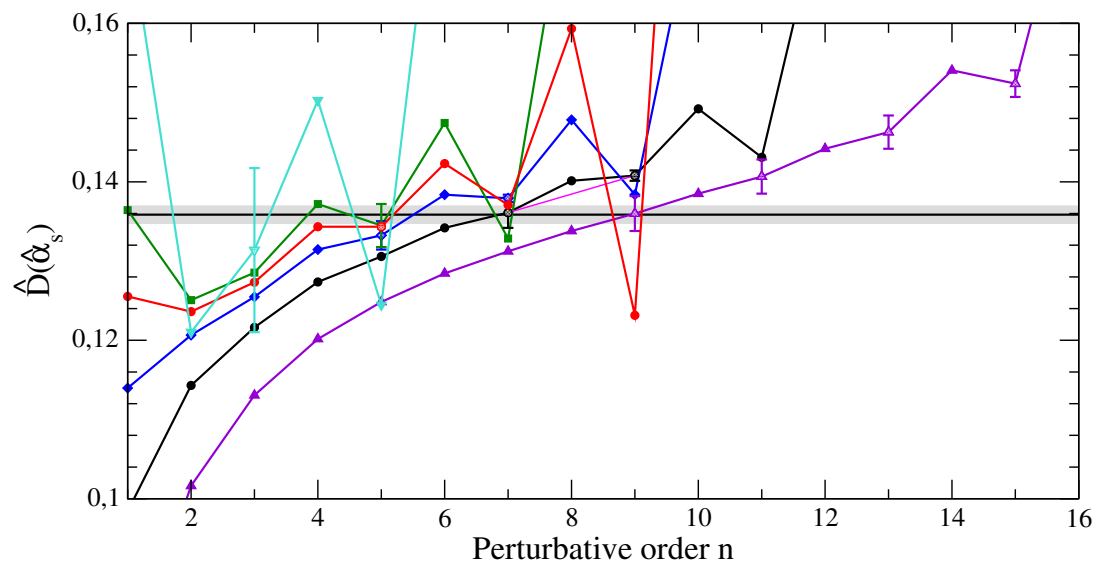

Figure 3. Partial sums up to order $n$ of the perturbative series for $\hat{D}\left(\hat{\alpha}_{s}\right)$ for different values of $C$ : $C=1$ (violet); $C=0$ (black); $C=-0.5$ (blue); $C=-0.783$ (red); $C=-1$ (green); $C=-1.5$ (cyan). The grey band corresponds to the Borel sum according to the central model of ref. [18]. For further details and discussion see the text.

To conclude this section, let us investigate the behaviour of the perturbative series for the Adler function $\hat{D}\left(\hat{\alpha}_{s}\right)$ order by order. To this end, in figure 3, we display the partial sums up to order $n$ for different values of the scheme parameter $C$. The colour coding is as follows: $\mathrm{C}=1$ (violet); $\mathrm{C}=0$ (black); $\mathrm{C}=-0.5$ (blue); $\mathrm{C}=-0.783$ (red); $\mathrm{C}=-1$ (green); $\mathrm{C}=-1.5$ (cyan). Perturbative orders higher than 5 have been taken according to the central model for the Borel-transformed Adler function of ref. [18], expressed in the coupling $\hat{\alpha}_{s}$. Furthermore, the grey band corresponds to the Borel sum also according to this model. 
We make the following observations: at low orders, the series is dominated by the infrared (IR) renormalons which for $\mathrm{C}=0$ yield a fixed sign series. (At $C<0$, as can be seen from the figure, this need no longer be the case.) At larger order the ultraviolet (UV) renormalons, being sign alternating, take over, and ultimately, the leading UV renormalon at $u=-1$ dominates. If $C$ becomes smaller and smaller, the dominance of UV renormalons starts at lower and lower orders and the general behaviour of the series becomes worse. As the shaded symbols with error bars, we denote small terms in the series. The error bar indicates the size of the respective term. For a strictly sign-alternating series, the smallest term would correspond to the closest approach of the series to the full result. Because we have fixed-sign and alternating-sign components in our series, we find terms accidentally small, and sometimes smaller than the small terms which are close to the Borel sum. In addition, for smaller $C$, the smallest term appears at lower orders, but also its size increases (and so would the corresponding error estimate). In the future, we plan to exploit the $C$ dependence of the Adler function series in $\hat{\alpha}_{s}$, in order to tune the smallest term to the presently available number of orders and to obtain more reliable error estimates.

\section{The total tau hadronic width}

We now turn our attention to the perturbative expansion for the total $\tau$ hadronic width. The central observable is the ratio $R_{\tau}$ for the total hadronic branching fraction to the electron branching fraction. It can be expressed as

$$
R_{\tau}=3 S_{\mathrm{EW}}\left(\left|V_{u d}\right|^{2}+\left|V_{u s}\right|^{2}\right)\left(1+\delta^{(0)}+\cdots\right),
$$

where $S_{\mathrm{EW}}$ is an electroweak correction and $V_{u d}$ as well as $V_{u s}$ CKM matrix elements. The perturbative QCD contribution is contained in $\delta^{(0)}$ and the ellipsis indicate further small subleading corrections. (See refs. [5, 18] for details.) For $\delta^{(0)}$ a complication arises, because it is calculated from a contour integral in the complex energy plane. On the other hand, we seek to resum the scale logarithms $\ln (Q / \mu)$, and the perturbative prediction depends on whether those logs are resummed after or before performing the contour integration. The first choice is called fixed-order perturbation theory (FOPT) and the second contour-improved perturbation theory (CIPT) [19].

In FOPT, the perturbative series of $\delta^{(0)}\left(a_{Q}\right)$ in terms of the $\overline{\mathrm{MS}}$ coupling $a_{Q}$ reads $[6,18]$

$$
\delta_{\mathrm{FO}}^{(0)}\left(a_{Q}\right)=a_{Q}+5.202 a_{Q}^{2}+26.37 a_{Q}^{3}+127.1 a_{Q}^{4}+\ldots
$$

On the other hand, in the $C$-scheme coupling $\hat{a}_{Q}$, the expansion for $\delta^{(0)}\left(\hat{a}_{Q}\right)$ is given by

$$
\begin{aligned}
\delta_{\mathrm{FO}}^{(0)}\left(\hat{a}_{Q}\right)= & \hat{a}_{Q}+(5.202+2.25 C) \hat{a}_{Q}^{2}+\left(27.68+27.41 C+5.063 C^{2}\right) \hat{a}_{Q}^{3} \\
& +\left(148.4+235.5 C+101.5 C^{2}+11.39 C^{3}\right) \hat{a}_{Q}^{4}+\ldots
\end{aligned}
$$

In Fig. $4, \delta_{\mathrm{FO}}^{(0)}\left(\hat{a}_{Q}\right)$ is displayed as a function of $C$. Assuming $c_{5,1}=283$, the yellow band again corresponds to removing or doubling the $O\left(\hat{a}^{5}\right)$ term. As for $\hat{D}(\hat{a})$, a nice plateau is found for $C \approx-1$. Taking $c_{5,1}=566$ and then doubling the $O\left(\hat{a}^{5}\right)$ results in the blue curve which does not show stability. Hence, this scenario again is disfavoured. In the red dots, which are located at $C=-0.882$ and $C=-1.629$, the $O\left(\hat{a}^{5}\right)$ correction vanishes, and the $O\left(\hat{a}^{4}\right)$ term is taken as the uncertainty. The point to the right has a substantially smaller error, and yields

$$
\delta_{\mathrm{FO}}^{(0)}\left(\hat{a}_{M_{\tau}}, C=-0.882\right)=0.2047 \pm 0.0034 \pm 0.0133 .
$$

Like for the Adler function, the second error covers the parametric uncertainty for $\alpha_{s}\left(M_{\tau}\right)$. In this case, the direct $\overline{\mathrm{MS}}$ prediction of eq. (20) is found to be

$$
\delta_{\mathrm{FO}}^{(0)}\left(a_{M_{\tau}}\right)=0.1991 \pm 0.0061 \pm 0.0119 .
$$




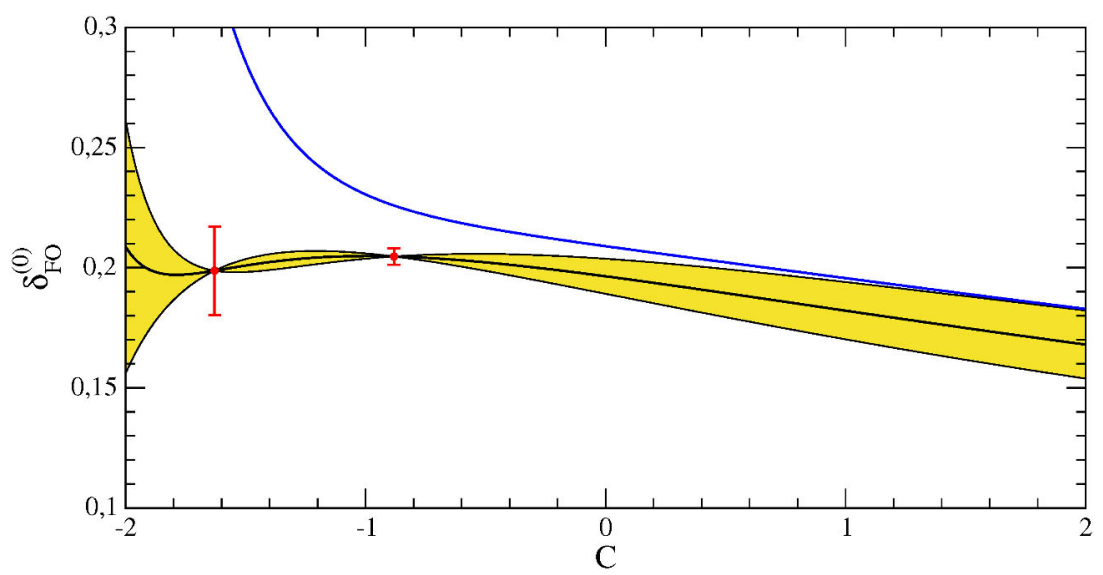

Figure 4. $\delta_{\mathrm{FO}}^{(0)}\left(\hat{a}_{Q}\right)$ of eq. (21) as a function of $C$. The yellow band arises from either removing or doubling the fifth-order term. In the red dots, the $O\left(\hat{a}^{5}\right)$ vanishes, and $O\left(\hat{a}^{4}\right)$ is taken as the uncertainty. For further explanation, see the text.

This value is somewhat lower, but within $1 \sigma$ of the higher-order uncertainty. Comparing, on the other hand, to the Borel model (BM) result of [18], which is given by

$$
\delta_{\mathrm{BM}}^{(0)}\left(a_{M_{\tau}}\right)=0.2047 \pm 0.0029 \pm 0.0130,
$$

it is found that (22) and (24) are surprisingly similar. In both cases, the $\alpha_{s}$ uncertainty is substantially larger than the higher-order one - especially given the recent increase in the $\alpha_{s}$ uncertainty provided by the PDG [17] - which underlines the good potential of $\alpha_{s}$ extractions from hadronic $\tau$ decays.

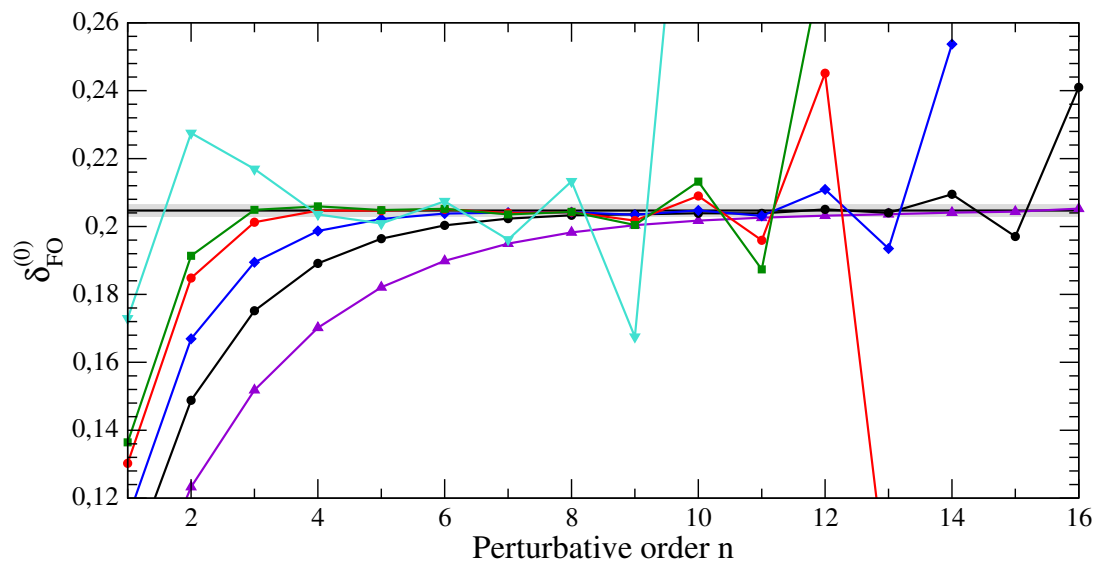

Figure 5. Partial sums up to order $n$ of the perturbative series for $\delta_{\mathrm{FO}}^{(0)}\left(\hat{a}_{M_{\tau}}\right)$ for different values of $C$ : $C=1$ (violet); $C=0$ (black); $C=-0.5$ (blue); $C=-0.882$ (red); $C=-1$ (green); $C=-1.5$ (cyan). The grey band corresponds to the Borel sum according to the central model of ref. [18]. For further discussion see the text.

In figure 5, we again provide partial sums up to order $n$ of the perturbative series for $\delta_{\mathrm{FO}}^{(0)}\left(\hat{a}_{M_{\tau}}\right)$ at different values of $C$. The colour coding is equivalent to figure 3: $C=1$ (violet); $C=0$ (black); 
$C=-0.5$ (blue); $C=-0.882$ (red); $C=-1$ (green); $C=-1.5$ (cyan). The $C=0$ series is close to the corresponding one in the $\overline{\mathrm{MS}}$ scheme. Although the general perturbative behaviour is similar to the Adler function, one observes that for $\delta_{\mathrm{FO}}^{(0)}\left(\hat{a}_{M_{\tau}}\right)$ it is substantially smoother. This is due to a suppression of the leading IR renormalon pole related to the gluon condensate. For larger $C$, the onset of the asymptotic behaviour is delayed, however the series needs more terms to approach the resummed result. For smaller $C$, and this is the case in particular for $C \approx-1$, the onset of the asymptotic behaviour is earlier, but also the series requires less terms to come close to the "true" value. In the region around $C=-0.882$ (red and green curves), a satisfactory approach to the Borel sum is achieved with just the four analytically available orders. If $C$ is still taken to be smaller, the behaviour of the series deteriorates, and error estimates from small terms become larger.

In CIPT, contour integrals over the running coupling, eq. (8), have to be computed, and hence the result cannot be given in analytical form. Graphically, as a function of $C, \delta_{\mathrm{CI}}^{(0)}\left(a_{M_{\tau}}\right)$ is displayed in Fig. 6. The general behavior is very similar to FOPT, with the exception that now also for $c_{5,1}=566$ a zero of the $O\left(\hat{a}^{5}\right)$ term is found. This time, both zeros have similar uncertainties, and employing the point with smaller error (in blue) results in

$$
\delta_{\mathrm{CI}}^{(0)}\left(\hat{a}_{M_{\tau}}, C=-1.246\right)=0.1840 \pm 0.0062 \pm 0.0084 .
$$

As has been discussed many times in the past (see e.g. [18]) the CIPT result lies substantially below the FOPT prediction, especially the $C$-scheme ones, and the Borel model. On the other hand, the parametric $\alpha_{s}$ uncertainty in CIPT turns out to be smaller.

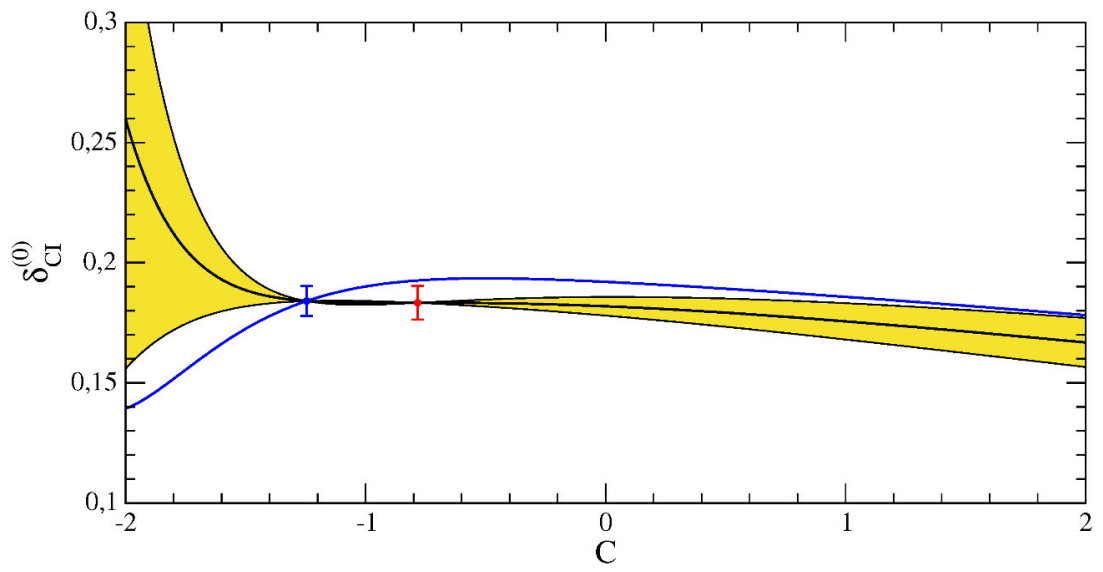

Figure 6. $\delta_{\mathrm{CI}}^{(0)}\left(\hat{a}_{Q}\right)$ as a function of $C$. The yellow band arises from either removing or doubling the fifth-order term. In the red and blue dots, the $O\left(\hat{a}^{5}\right)$ vanishes, and $O\left(\hat{a}^{4}\right)$ is taken as the uncertainty. For further explanation, see the text.

\section{Conclusions}

In ref. [3], see eq. (6), we have defined a class of QCD couplings $\hat{a}_{Q}$, such that the scale running is explicitly scheme invariant, and scheme changes can be parameterised by a single constant $C$. For this reason, we have termed $\hat{a}_{Q}$ the $C$-scheme coupling. Scheme transformations correspond to shifts in the QCD scale $\Lambda$. It is furthermore seen that $C$ changes are also governed by the $\beta$-function $\hat{\beta}\left(\hat{a}_{Q}\right)$ and hence scale and scheme transformations are equivalent. 
We have applied the coupling $\hat{a}_{Q}$ to investigations of the perturbative series of the reduced Adler function $\hat{D}$. Our central result is given in eq. (16). Its higher-order uncertainty turned out larger than the corresponding $\overline{\mathrm{MS}}$ prediction (17), but we consider (16) to be more conservative and realistic.

We also studied the perturbative expansion of the $\tau$ hadronic width, employing the coupling $\hat{a}_{Q}$. In this case our central prediction in FOPT is given in eq. (22). Surprisingly, the result (22) is found very close to the prediction (24) of the central Borel model developed in ref. [18], hence providing some support for this approach.

The disparity between FOPT and CIPT predictions for $\delta^{(0)}$ is not resolved by the $C$-scheme. As is seen from eq. (25) and figure 6, the CIPT result turns out substantially lower (as is the case for the $\overline{\mathrm{MS}}$ prediction). This suggests to return to detailed investigations of Borel models, this time expressed in the $C$-scheme coupling $\hat{a}$, in order to investigate the scheme dependence of such models. This could result in an improved extraction of $\alpha_{s}$ from hadronic decays of the $\tau$ lepton.

\section{Acknowledgments}

Helpful discussions with Martin Beneke are gratefully acknowledged. The work of MJ and RM has been supported in part by MINECO Grant number CICYT-FEDER-FPA2014-55613-P, by the Severo Ochoa excellence program of MINECO, Grant SO-2012-0234, and Secretaria d'Universitats i Recerca del Departament d'Economia i Coneixement de la Generalitat de Catalunya under Grant 2014 SGR 1450. DB's is supported by the São Paulo Research Foundation (FAPESP) grant 15/20689-9, and by CNPq grant 305431/2015-3.

\section{References}

[1] G. 't Hooft and M.J.G. Veltman, Nucl. Phys. B 44 (1972) 189.

[2] W.A. Bardeen, A.J. Buras, D.W. Duke and T. Muta, Phys. Rev. D 18 (1978) 3998.

[3] D. Boito, M. Jamin and R. Miravitllas, Phys. Rev. Lett. 117 (2016) 152001, arXiv:1606.06175 [hep-ph].

[4] S.L. Adler Phys. Rev. D 10 (1974) 3714.

[5] E. Braaten, S. Narison and A. Pich Nucl. Phys. B 373 (1992) 581.

[6] P.A. Baikov and K.G. Chetyrkin and J.H. Kühn, Phys. Rev. Lett. 101 (2008) 012002, arXiv:0801.1821 [hep-ph].

[7] M. Jamin and R. Miravitllas, JHEP 1610 (2016) 59, arXiv:1606.06166 [hep-ph].

[8] S.J. Brodsky, P.G. Lepage and P.B. Mackenzie, Phys. Rev. D 28 (1983) 228.

[9] S.J. Brodsky and Xing-Gang Wu, Phys. Rev. Lett. 109 (2012) 042002, arXiv:1203.5312 [hep-ph].

[10] M. Mojaza, S.J. Brodsky and Xing-Gang Wu, Phys. Rev. Lett. 110 (2013) 192001, arXiv:1212.0049 [hep-ph].

[11] G. Grunberg, Phys. Rev. D 29 (1984) 2315.

[12] T. van Ritbergen, J.A.M. Vermaseren and S.A. Larin, Phys. Lett. B 400 (1997) 379, hep$\mathrm{ph} / 9701390$.

[13] P.A. Baikov, K.G. Chetyrkin and J.H. Kühn, arXiv:1606.08659 [hep-ph].

[14] W. Celmaster and R.J. Gonsalves, Phys. Rev. D 20 (1979) 1420.

[15] L.S. Brown, L.G. Yaffe and C.-X. Zhai, Phys. Rev. D 46 (1992) 4712, hep-ph/9205213.

[16] M. Beneke, PhD Thesis, Munich, 1993.

[17] K.A. Olive and others (Particle Data Group), Chin. Phys. C, 38 (2014) 090001.

[18] M. Beneke and M. Jamin, JHEP 09 (2008) 044, arXiv:0806.3156 [hep-ph].

[19] F. Le Diberder and A. Pich, Phys. Lett. B 286 (1992) 147. 